\title{
Semi-automatic Catheter Reconstruction from Two Views
}

\author{
Matthias Hoffmann ${ }^{1, \star}$, Alexander Brost ${ }^{1}$, Carolin Jakob ${ }^{1}$, Felix Bourier ${ }^{2}$, \\ Martin Koch ${ }^{1}$, Klaus Kurzidim ${ }^{2}$, Joachim Hornegger ${ }^{1}$, and Norbert Strobel ${ }^{3}$ \\ 1 Pattern Recognition Lab., \\ Friedrich-Alexander-University Erlangen-Nuremberg, Erlangen, Germany \\ Matthias.Hoffmann@cs.fau.de \\ 2 Krankenhaus Barmherzige Brüder, Regensburg, Germany \\ 3 Siemens AG, Healthcare Sector, Forchheim, Germany
}

\begin{abstract}
We propose novel methods for (a) detection of a catheter in fluoroscopic images and (b) reconstruction of this catheter from two views. The novelty of (a) is a reduced user interaction and a higher accuracy. It requires only a single seed point on the catheter in the fluoroscopic image. Using this starting point, possible parts of the catheter are detected using a graph search. An evaluation of the detection using 66 clinical fluoroscopic images yielded an average error of $0.7 \mathrm{~mm} \pm 2.0$ $\mathrm{mm}$. The novelty of (b) is a better ability to deal with highly curved objects as it selects an optimal set of point correspondences from two point sequences describing the catheters in two fluoroscopic images. The selected correspondences are then used for computation of the 3-D reconstruction. The evaluation on 33 clinical biplane images yielded an average backprojection error of $0.4 \mathrm{~mm} \pm 0.6 \mathrm{~mm}$.
\end{abstract}

\section{Introduction}

Radio-frequency catheter ablation can be used to treat atrial fibrillation, currently being the most common heart arrhythmia. Ablation is usually performed under fluoroscopic guidance using a C-arm system. Unfortunately, in many cases physicians can only see the catheters, while the left atrium remains invisible. Often a 3-D overlay is used to outline the structure of the heart [6]. In contrast to electro-anatomical mapping systems, $\mathrm{C}$-arm systems provide only 2 -D projection images but no direct 3-D information of the catheters. Physicians need to derive the respective catheter positions from fluoroscopic imaging mentally. Currently, point localization from two views is the only way to verify a catheter position. Electro-anatomical mapping systems provide a 3-D visualization of the catheters inside a pre-operative data set. To provide the same functionality, we propose a novel semi-automatic method to reconstruct a catheter used in electrophysiology (EP) procedures from two views. In addition, multiple reconstructions of the mapping catheter at different pulmonary veins $(\mathrm{PVs})$ or at different positions at one PV can be used for registration. Previously, a method for initial registration using the catheter in the coronary sinus was proposed [5]. Our proposed

\footnotetext{
* Corresponding author.
} 
method would help to use these methods in clinical practice. Furthermore, the 3$\mathrm{D}$ catheter model could be used as input for other algorithms used for catheter tracking 2] or motion compensation [4]. Previous methods dealed only with guide wires or catheters with low curvature [310]. Blob detection can be used to detect the electrodes of the catheter, but not its entirety [8]. Our novel method provides the capability to achieve a 3-D reconstruction of a complex catheter such as the circumferential mapping catheter by requiring only minimal user input. User interaction is limited to a single click on the catheter in each of the imaging frames. This contribution is structured as follows. In the second section, details on the catheter detection are provided. In the third section, the catheter reconstruction is presented. Our evaluation and results are presented in section four. In the final section, we discuss our results and draw some conclusions.

\section{Catheter Detection}

Catheter detection is performed in three steps: (i) a seedpoint on the transition from the shaft to the catheter tip is marked. Based on image pre-processing, possible parts of the catheter are identified, and (ii) combined to a graph. (iii) a set of edges of the graph is selected. This provides us with a final estimate for the overall catheter. In step (i), a medialness filter [9] is applied to the image. Then a binary image is generated using a dynamic threshold. Before the skeletonization of the binary image is computed, an opening and closing operation is performed to close small gaps. The search space for the catheter is then reduced by selecting the 150,000 pixels from the $1,024 \times 1,024$-image that are nearest to the seedpoint and the skeleton. The skeleton contains the parts of the image which belong most likely to the catheter. In step (ii), the skeleton is transformed into a graph to obtain an analytical representation for a catheter model. The edges of the graph are point sequences that basically follow the skeleton. The nodes of this graph are the points where a point sequence starts or meets another point sequence. An example of such a graph is given in Fig. 1(b). For the computation of this graph, a cost function is defined using the filtered image and a distance transformation of the skeleton. Using this cost function, the shortest paths from all pixels to the seedpoint are computed by Dijkstra's algorithm. The circles and lines of the skeleton are then integrated in the graph. Since the skeleton is not continuous but may contain gaps, the above computed shortest paths from certain feature points to the seedpoint are used as edges for the new graph. The feature points are computed such that the shortest paths from these points to the seedpoint form the circles and lines of the skeleton as well as certain additional connections between parts of the skeleton. For the circles of the skeleton, the two points on the circle which are farthest away from the seedpoint are used as feature points. These points can easily be found by searching for points on the skeleton which have only neighboring skeleton pixels with a lower distance to the seedpoint, see Fig. 1 The shortest paths from these points to the seed point will either run in clockwise or counterclockwise direction, respectively. The remaining lines of the skeleton are inserted into the graph by including the edges belonging 


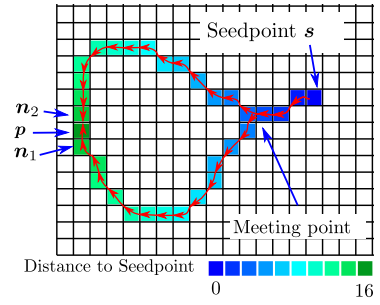

(a)

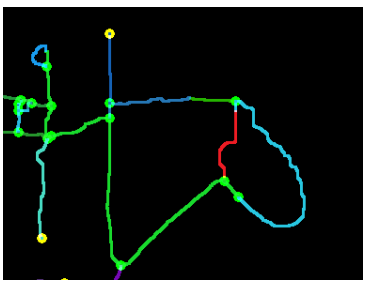

(b)

Fig. 1. (a) Circles can be found by considering pixels at which two paths from different directions meet. The color indicates the distance to the seedpoint. The two neighboring pixels of $\boldsymbol{p}$ are closer to the seedpoint than $\boldsymbol{p}$ itself. In such a case, two paths from different directions meet at pixel $\boldsymbol{p}$. By selecting $\boldsymbol{p}$ and $\boldsymbol{n}_{2}$ as feature points, the circle is given by their respective paths to $s$ (b) An example of the generated graph. Each path from a feature point to a node is given in a different color to make them distinguishable.

to the shortest paths from endpoints of the skeleton to the seedpoint. Finally, connections between parts of the skeleton are added to overcome gaps in the skeleton. The connections are found similarly to the circles but this time the points with higher distance to all neighboring points do not need to be part of the skeleton. For each connection, the distance $l_{\mathrm{b}}$ in pixels to the existing graph is computed as well as the distance $l_{\mathrm{c}}$ to the meeting point. Finally, the endpoints of edges in the graph which are neighbored in the image are connected such that circles arise. In step (iii), the above computed graph serves as search space for the catheter. With the seedpoint, some information is given by the physician. The search for the catheter is split up into the search for the catheter shaft and the catheter tip. Due to the standard setups of the C-arm used in electrophysiology procedures, prior knowledge of the image orientation can be taken into account. The shaft of the catheter is considered to enter the image at the bottom edge. Therefore all shortest paths on the graph from the seedpoint to points in the lower half of the image are considered as candidates for the shaft. They are examined with respect to their length, curvature, direction and ending angle. The best shaft candidate maximizes the length while minimizing the curvature. Its direction as well as its ending angle are as vertical as possible. The elliptical catheter tip is determined by considering all possible paths starting from the seedpoint that were not used for the shaft. For selecting the best path, an ellipse is fitted to the path and the ellipse that approximates the elliptical part best is chosen, according to their response to the medialness filter. We also try to make sure that the shape of the path is as elliptical as possible. This is measured by considering the average distance from the ellipse to the path and vice versa. Additionally, the size of the approximated ellipse is used to reject candidates for which the ellipse is not plausible with respect to the known catheter shape. Since the search space for the ellipse search is very large, a population based search strategy is used [1]. In this strategy, an initial population of short paths starting from the seedpoint is evaluated. In each iteration the offspring which continues the paths from the previous generation is evaluated as described above. If a 


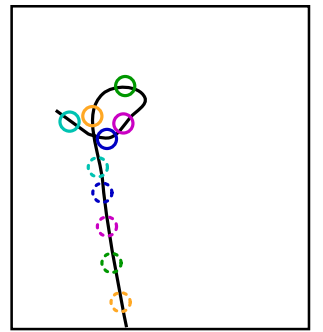

(a)

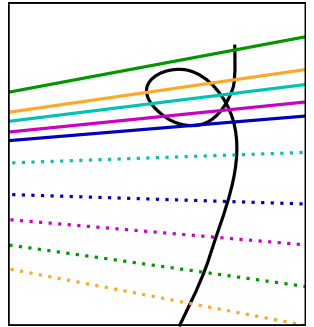

(b)

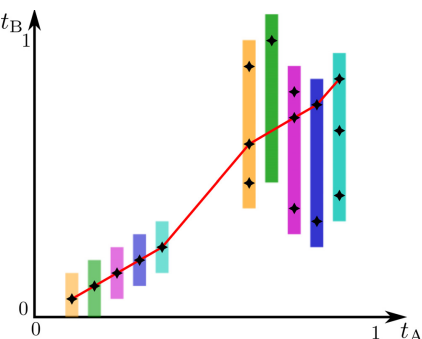

(c)

Fig. 2. The spline in (a) is sampled and for each sampling point, possible point correspondences are compute by intersecting the epipolar line with the spline in (b). For each possible point correspondence, the spline parameters are stored (c). A correspondence function that computes for each spline point $C_{A}\left(t_{A}\right)$ the corresponding spline point $C_{B}\left(t_{B}\right)$ has to be monotonic. The optimal correspondence function interpolates as much as possible correspondence candidates.

population limit is reached, the paths with the worst fitness value drop out of the population. The search stops, if in the previous three generations no better solution was found. The final result is a 2-D spline along the detected catheter.

\section{Catheter Reconstruction}

The catheter is reconstructed in 3-D from the two 2-D splines, $C_{A}\left(t_{A}\right)$ and $C_{B}\left(t_{B}\right)$, in image plane $\mathrm{A}$ and $\mathrm{B}$. They run from bottom to top, i.e. $C_{A}(0)$ denotes the lowest point at the shaft, $C_{A}(1)$ is the last point at the tip. Reconstruction is performed by triangulation of corresponding points. To find them, $C_{A}\left(t_{A}\right)$ is equidistantly sampled and for each point, potential corresponding points are computed by intersecting the epipolar line with the spline $C_{B}\left(t_{B}\right)$ in image B. For objects with high curvature, a point in A might yield multiple intersections in B. So, a strategy for selecting one of those intersections has to be established. Due to inaccuracies in a practical scenario, the epipolar line might intersect the spline not at the actual corresponding point. As shown by the solid green line in Fig. 2, intersections can, however, occur at other points. A greedy selection strategy as proposed by Baert et al. 2] might yield wrong results in such a case. It selects the intersection point next to the previous one as corresponding point. If one point would be chosen incorrectly, all subsequent points would be incorrect as well, due to the linearity constraint. To avoid such a problem, the selection process is performed after the computation of all correspondence candidates. This is achieved by computing an optimal correspondence function $f: t_{A} \rightarrow t_{B}$ that takes all possible point correspondences $\left(t_{A}, t_{B}\right)$ into account. It should map the first spline parameter to the second and be able to deal with missing points. The monotonic function that contains as many as possible correspondence pairs is selected, see Fig.2. It might be necessary to skip some points in order to include following points with regard to the monotony constraint. An estimate of missing correspondence points can be computed by interpolating $f$. The computation 


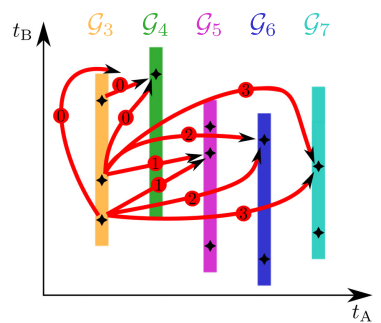

(a)

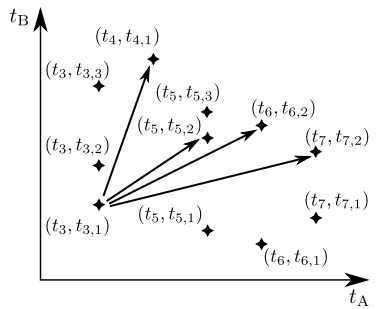

(b)

Fig. 3. (a) The optimal correspondence function can be found by computing a shortest path in a graph. The nodes of the graph are the correspondence candidates and the edges connect them such that monotony constraint is preserved. The cost of an edge is equal to the number of skipped points. (b) An example of the graph, the edges are visualized only for node $\left(t_{3}, t_{3,1}\right)$.

of this function can be considered as a shortest path problem. A node of the graph corresponds to a possible point correspondence pairs $\left(t_{i}, t_{i, j}\right) . t_{i}$ denotes the spline parameter of the $i$-th sample point of $C_{A}$. For a fixed point $C_{A}\left(t_{i}\right)$, the possible corresponding points are then given by $C_{B}\left(t_{i, 1}\right), \ldots, C_{B}\left(t_{i, m}\right)$, assuming $m$ intersection points with the spline in image plane B. All nodes with the same index $i$ form a group $\mathcal{G}_{i}$. The edges are designed such that the monotony constraint is preserved. The node $\left(t_{i}, t_{i, j}\right)$ is connected by a directed edge to a single node of each group $\mathcal{G}_{i+l}$ with a group jump $l>0$. The target node of the edge is the node with the smallest index $t_{i+l, k}$ that fulfills the constraint $t_{i+l, k}>t_{i, j}$. Including an edge from group $\mathcal{G}_{i}$ to a group $\mathcal{G}_{i+l}, l \geq 2$ means that the possible point correspondences for the sample points in between are neglected. As only as few points as possible should be omitted, skipping a group is penalized by a cost of 1 , so the cost of an edge is $l-1$.

\section{Evaluation and Results}

For the evaluation of the detection, clinical data consisting of 33 biplane sequences from 27 patients were used. For each of the resulting 66 monoplane sequences, a detection was computed and compared with a gold standard segmentation. The error was measured as the distance from detected catheter pixels to the gold standard center line. For the generation of the search graph, it turned out to be sufficient to include only those circles in the graph for which the piece from a feature point to the meeting point is longer than 75 pixels. For the connections between parts of the skeleton, taking only those with $l_{\mathrm{c}}>75$ pixels and $l_{\mathrm{c}} / l_{\mathrm{b}}>10$ was a good choice. The search for the catheter tip started with a path length of 100 pixel and stopped at latest if a length of 600 pixel was reached. A possible detection result is given in Fig. 4. For the whole catheter, the mean error of the detection is $0.7 \mathrm{~mm} \pm 2.0 \mathrm{~mm}$ and the min and max error is $0.0 \mathrm{~mm}$ and $19.9 \mathrm{~mm}$. The pixel spacing is $0.1725 \mathrm{~mm}$, so, the mean error corresponds to 4.1 pixels in the 2-D images. For the tip only, the mean error is $0.4 \mathrm{~mm} \pm 0.3 \mathrm{~mm}$, 


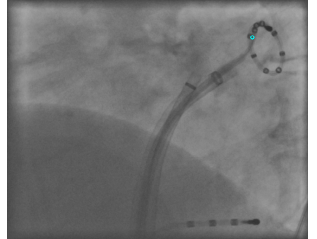

(a)

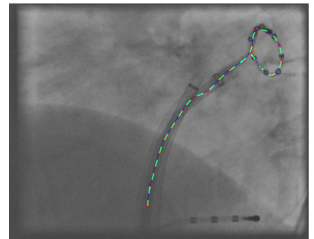

(b)

Fig. 4. (a) Manual initialization of the catheter detection. The seedpoint of the proposed method is set by the physician at the transition between the shaft and the catheter tip. (b) Resulting spline after detection of the catheter.

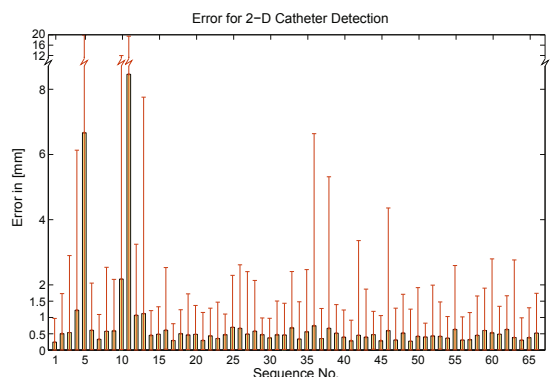

(a)

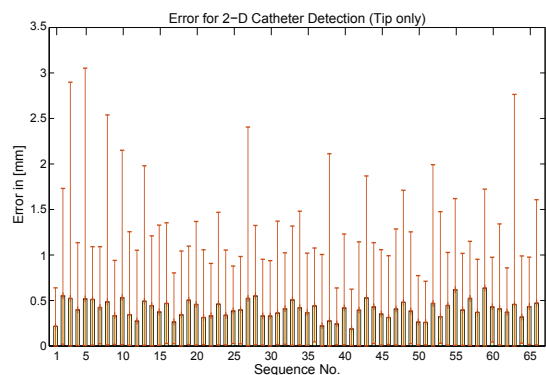

(b)

Fig. 5. Mean detection errors for each sequence, the thin bars denote the maximal and minimal error. In (a) the detection error for the whole catheter is given. The total mean error is $0.7 \mathrm{~mm} \pm 2.0 \mathrm{~mm}$. (b) shows the error for the catheter tip. The total mean error is $0.4 \mathrm{~mm} \pm 0.3 \mathrm{~mm}$.

the min and max error is $0.0 \mathrm{~mm}$ and $3.1 \mathrm{~mm}$, see Fig. 5 . As the catheter tip is the important part for the physician, our results meet current clinical requirements. The average percentage of undetected pixels was $6.0 \% \pm 10.2 \%$. The min and max percentage of undetected pixels were $0.0 \%$ and $44 \%$, respectively. The average percentage of false positive pixels was $1.3 \% \pm 7.1 \%$ The min and max percentage of false positives were $0.0 \%$ and $47 \%$, respectively. The evaluation of the reconstruction was performed using clinical data. Since no clinical 3-D data was available as ground truth, the result of the $3-\mathrm{D}$ reconstruction was backprojected into the image planes and the error of the reprojected points to a gold standard segmentation was computed. The resulting mean error was $0.4 \mathrm{~mm} \pm 0.6 \mathrm{~mm}$ and 2.3 pixels, respectively, for the whole catheter, and $0.7 \mathrm{~mm} \pm 0.7 \mathrm{~mm}$ for the catheter tip. The min and max error was $0.0 \mathrm{~mm}$ and $4.8 \mathrm{~mm}$ in both cases. Using the results of the detection for reconstruction, the result differed to the reconstruction using the gold-standard by $1.5 \mathrm{~mm} \pm 2.5 \mathrm{~mm}$, the $\min$ and $\max$ difference was $0.0 \mathrm{~mm}$ and $15.7 \mathrm{~mm}$. Fig. 6 shows the results for each biplane pair. The detection requires on average $8.7 \mathrm{sec}$ and the reconstruction $0.15 \mathrm{sec}$ on an Intel i7 with $2,67 \mathrm{GHz}$ and $4 \mathrm{~GB}$ RAM. The medialness filter computation was performed on a NVIDIA Quadro FX 880M GPU. 


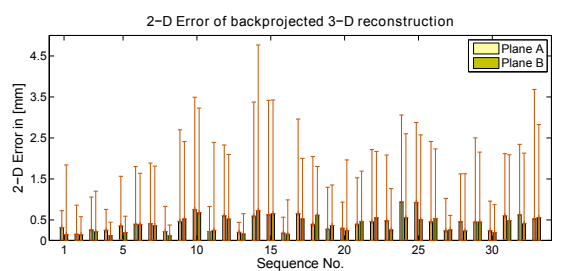

(a)

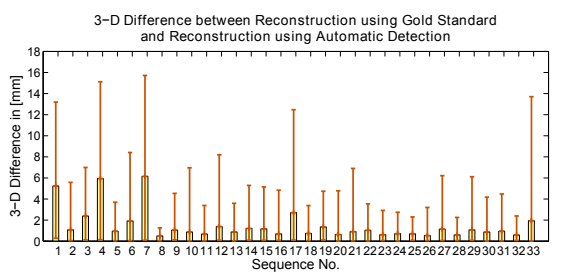

(b)

Fig. 6. (a) shows the mean error of the reprojected 3-D reconstruction for each sequence. The overall mean error is $0.4 \mathrm{~mm} \pm 0.6 \mathrm{~mm}$. (b) shows the deviation of the reconstruction using the detection results to the reconstruction using the gold standard segmentation. The mean deviation is $1.5 \mathrm{~mm} \pm 2.5 \mathrm{~mm}$.

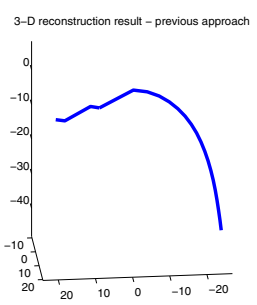

(a)

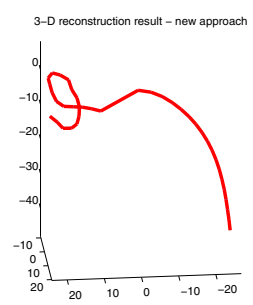

(b)

Fig. 7. Reconstruction result using the previous approach (a) and our new method (b). While both methods perform well for the linear part of the catheter, the previous approach fails for the curved catheter tip.

\section{Discussion and Conclusions}

The results of the catheter detection show that detection of the whole catheter is possible if only a single seedpoint is provided. The mean error is $0.7 \mathrm{~mm}$ and therefore sub-millimeter accuracy can be reached for catheter parts that were detected by the algorithm, thus, enabling catheter localization for EP applications 7]. The percentage of undetected catheter parts is with $6.0 \%$ lower than the methods proposed by Honnorath et al. [10] and Franken et al. [8]. Also the number of false positive pixels is lower when compared to [10]. Compared to the previous approach by Baert [2], our new approach can deal better with curved catheters as shown in Fig. 7. In some cases, two catheters may be overlapping and the wrong catheter is selected as shaft. In such cases, the physician could manually adjust the detection result before performing the 3 -D reconstruction.

Acknowledgments. This work was supported by the German Federal Ministry of Education and Research (BMBF) in the context of the initiative Spitzencluster Medical Valley, project grant Nos. 01EX1012A and 01EX1012E, respectively. Additional funding was provided by Siemens AG, Healthcare Sector. 


\section{References}

1. Bäck, T., Hammel, U., Schwefel, H.: Evolutionary computation: Comments on the history and current state. IEEE Tr. on Evolutionary Comp. 1(1), 3-17 (1997)

2. Baert, S., van de Kraats, E., van Walsum, T., Viergever, M., Niessen, W.: Threedimensional guide-wire reconstruction from biplane image sequences for integrated display in 3-D vasculature. IEEE Tr. on Medical Imaging 22(10), 1252-1258 (2003)

3. Bender, H.-J., Männer, R., Poliwoda, C., Roth, S., Walz, M.: Reconstruction of 3D catheter paths from 2D X-ray projections. In: Taylor, C., Colchester, A. (eds.) MICCAI 1999. LNCS, vol. 1679, pp. 981-989. Springer, Heidelberg (1999)

4. Brost, A., Liao, R., Strobel, N., Hornegger, J.: Respiratory motion compensation by model-based catheter tracking during EP procedures. Medical Image Analysis 14(5), 695-706 (2010)

5. Brost, A., Bourier, F., Yatziv, L., Koch, M., Hornegger, J., Strobel, N., Kurzidim, K.: First steps towards initial registration for electrophysiology procedures. In: Wong, K.H., Holmes III, D.R. (eds.) Medical Imaging 2011: Visualization, ImageGuided Procedures, and Modeling, SPIE, vol. 7964, p. 79641 (2011)

6. Dilling-Boer, D., van der Merwe, N., Adams, J., Foulon, S., Goethals, H., Willems, R., Ector, H., Heidbüchel, H.: Ablation of Focally Induced Atrial Fibrillation. Journal of Cardiovascular Electrophysiology 15(2), 200-205 (2004)

7. Esteghamatian, M., Azimifar, Z., Radau, P., Wright, G.: Real-time 2D-3D MR cardiac image registration during respiration using extended Kalman filter predictors. In: Baozong, Y. (ed.) 9th International Conference on Signal Processing, ICSP 2008, pp. 1325-1328. IEEE (2008)

8. Franken, E., Rongen, P., van Almsick, M., ter Haar Romeny, B.: Detection of Electrophysiology Catheters in Noisy Fluoroscopy Images. In: Larsen, R., Nielsen, M., Sporring, J. (eds.) MICCAI 2006, Part II. LNCS, vol. 4191, pp. 25-32. Springer, Heidelberg (2006)

9. Gülsün, M., Tek, H.: Robust Vessel Tree Modeling. In: Metaxas, D., Axel, L., Fichtinger, G., Székely, G. (eds.) MICCAI 2008, Part I. LNCS, vol. 5241, pp. 602611. Springer, Heidelberg (2008)

10. Honnorat, N., Vaillant, R., Paragios, N.: Guide-Wire Extraction through Perceptual Organization of Local Segments in Fluoroscopic Images. In: Jiang, T., Navab, N., Pluim, J.P.W., Viergever, M.A. (eds.) MICCAI 2010, Part III. LNCS, vol. 6363, pp. 440-448. Springer, Heidelberg (2010) 